\title{
Patient Reported Outcomes Version of CTCAE
}

National Cancer Institute

\section{Source}

National Cancer Institute. Patient Reported Outcomes Version of CT CAE. NCI Thesaurus.

Code C103843.

A library of items for patient self-reporting of symptoms and side effects associated with cancer treatment trials. 